\title{
Substrate-adsorbate coupling in CO-adsorbed copper
}

\author{
Steven P. Lewis and Andrew M. Rappe \\ Department of Chemistry and Laboratory for Research on the Structure of Matter, \\ University of Pennsylvania, Philadelphia, PA 19104.
}

(June 9, 2021)

\begin{abstract}
The vibrational properties of carbon monoxide adsorbed to the copper (100) surface are explored within density functional theory. Atoms of the substrate and adsorbate are treated on an equal footing in order to examine the effect of substrate-adsorbate coupling. This coupling is found to have a significant effect on the vibrational modes, particularly the in-plane frustrated translation, which mixes strongly with substrate phonons and broadens into a resonance. The predicted lifetime due to this harmonic decay mechanism is in excellent quantitative agreement with experiment.
\end{abstract}

An important consequence of molecular adsorption to a metal surface is the emergence of new, low-frequency vibrations associated with fluctuations of the chemisorption bond. These "external modes" correspond to translations and rotations of the free molecule which become frustrated upon adsorption to a substrate. They play an instrumental role in many fundamental processes at surfaces, including chemical reactivity, desorption, and surface diffusion [1] 3], and are, therefore, of intense scientific interest.

In this letter, we theoretically investigate the vibrational dynamics of in-plane frustrated translational (FT) motion for a prototypical adsorbed system: carbon monoxide on the (100) surface of copper at halfmonolayer coverage. Frustrated translational vibrations are considered particularly important in surface chemistry because they are typically very low in energy (a few meV) and are thus thermally activated. This mode has been found to be extremely short-lived for $\mathrm{CO}$ on copper, with a lifetime in the few picosecond range [4 6]. The mechanisms governing the relaxation of this mode are of considerable interest.

The present study focuses on the role of the substrate lattice in FT decay. Vibrational states for the combined substrate-adsorbate system are computed from first principles, with all atoms treated on an equal footing. These investigations reveal that the dominant contribution to FT relaxation comes from resonant mixing of frustrated translations with long-wavelength bulk copper phonons. This is a purely harmonic mechanism, and it gives rise to a computed lifetime in good quantitative agreement with experiment [4]. To our knowledge, this is the first detailed, quantum-mechanical investigation for an adsorbed metal system that demonstrates the strong interaction between adsorbate motion and long-wavelength bulk phonons. An earlier classical model of FT damping [7] considered this type of coupling for the case of an isolated FT oscillator attached to a semi-infinite, elastic medium. The damping was expressed as a macroscopic frictional force. Other microscopic theoretical studies have primarily considered only anharmonic effects in ad- dressing adsorbate-lattice vibrational coupling, and have failed to identify this resonance because they have not modeled a large enough portion of the substrate to accommodate the long-wavelength phonons involved in resonant harmonic mixing.

Carbon monoxide on the (100) surface of copper has been the subject of a recent pump-probe laser experiment [4] to investigate the role that FT motion plays in energy transfer between the substrate and the adsorbate overlayer. This study measures the time-resolved vibrational response of the $\mathrm{CO}$ molecules to picosecond heating of the copper substrate, and interprets the resulting transient-response data as revealing the vibrational dynamics of FT motion. This experiment observes a rapid vibrational excitation followed by a slower decay with a characteristic damping time of $2.3 \pm 0.4 \mathrm{ps}$. The authors model the vibrational relaxation by a two heatbath model coupling adsorbate motion to electrons and phonons of the substrate. The fitted relaxation times due to electronic and lattice coupling are $5.1 \pm 0.4$ ps and $4.2 \pm 0.7 \mathrm{ps}$, respectively. These measurements were taken at a substrate temperature of about $100 \mathrm{~K}$ and at a surface coverage of half of a monolayer.

Vibrational damping in this system has also been addressed theoretically. A recent quantum chemistry analysis 8] has examined relaxation of the adsorbate vibrational motions through nonadiabatic coupling to conduction electrons of the substrate. In this study, the copper substrate is modeled as a cluster with atoms fixed in their experimental positions. The coupling rates to the copper conduction electrons are determined using the Fermi golden rule, and the FT mode is found to decay slowly via this mechanism $(\tau=108 \mathrm{ps})$ compared to other modes, such as the frustrated rotation (FR) and the internal $\mathrm{CO}$ stretch vibration $(\tau=2-3 \mathrm{ps})$. The same authors have also performed a classical molecular-dynamics simulation of this system to include both vibrational and electronic damping mechanisms [9]. Nonadiabatic electronic coupling is included via an electronic friction formalism, and the vibrational motion is described by empirical Morse potentials. The substrate is modeled as a 
TABLE I. Computed equilibrium layer separations for (a) CO adsorbed to the copper (100) surface, (b) the bare copper (100) surface, and (c) a free CO molecule. The comparison to bulk copper refers to the computed separation between (100) layers in bulk copper: $1.796 \AA$.

\begin{tabular}{|c|c|c|c|c|}
\hline Layer & \multicolumn{2}{|c|}{ Separation $(\AA)$} & $\%$ difference from & Experiment \\
\hline Pairs & Covered Site & Empty Site & bulk Cu spacing & \\
\hline
\end{tabular}

(a) CO on copper (100)

\begin{tabular}{|c|c|c|c|}
\hline $\mathrm{O}-\mathrm{C}$ & 1.138 & & $1.15 \pm 0.10 \AA^{\mathrm{a}}$ \\
\hline $\mathrm{C}-\mathrm{Cu}_{1}$ & 1.852 & & $1.92 \pm 0.05 \AA^{\mathrm{a}}$ \\
\hline $\mathrm{Cu}_{1}-\mathrm{Cu}_{2}$ & 1.805 & 1.768 & \\
\hline $\mathrm{Cu}_{2}-\mathrm{Cu}_{3}$ & 1.804 & 1.821 & \\
\hline $\mathrm{Cu}_{3}-\mathrm{Cu}_{4}$ & 1.809 & 1.792 & \\
\hline
\end{tabular}

(b) Bare copper (100)

$\begin{array}{lrcc}\mathrm{Cu}_{1}-\mathrm{Cu}_{2} & 1.771 & -1.38 & -(1.2-2.4) \%^{\mathrm{b}} \\ \mathrm{Cu}_{2}-\mathrm{Cu}_{3} & 1.807 & +0.60 & +(0.0-1.0)^{\mathrm{b}} \\ \mathrm{Cu}_{3}-\mathrm{Cu}_{4} & 1.795 & -0.02 & \end{array}$

(c) Free CO molecule

$\mathrm{O}-\mathrm{C}$

${ }^{\text {a }}$ Reference $\sqrt{14}$

${ }^{\mathrm{b}}$ Reference 15

three-layer copper slab. These calculations again predict a comparatively slow $\mathrm{FT}$ decay rate $(\tau=14 \mathrm{ps})$ at low temperatures. As temperature increases above about 150 $\mathrm{K}$, however, the computed decay time does enter the few picosecond range.

The vibrational properties of molecules interacting with surfaces have generally been described in terms of localized vibrational modes involving only motion of the molecule relative to the surface. Atoms of the substrate are typically frozen in place, and the vibrational modes, by construction, only span the atomic degrees of freedom of the adsorbate. In this theoretical study, however, we are explicitly interested in exploring the nature of the coupling between molecular and substrate vibrations, and thus we require an unbiased theory which incorporates atomic motions of both components equally. This is accomplished by computing interatomic harmonic force constants for the combined system, and solving the resulting coupled-oscillator equations to get the normal modes of vibration.

For the purposes of computing force constants, the adsorbed surface is modeled as a seven-layer copper (100) slab with half-monolayer CO coverage of the slab surfaces in a $(\sqrt{2} \times \sqrt{2}) R 45^{\circ}$ pattern. Full periodicity is imposed by periodically repeating the slab perpendicular to the surface with $12 \AA$ of vacuum separating adjacent slabs. Force constants are obtained by making a series of perturbations of the system away from its equilibrium configuration, and computing the resulting forces on all of the atoms, from first principles. Long-wavelength bulk cop- per phonons are included in the calculation by defining a much thicker effective slab [10], in which 1000 bulk-like layers are inserted in the middle of the seven-layer slab. Interlayer force constants for bulk copper are computed once in a separate calculation, and thus little additional computational effort is required to set up the coupledoscillator equations for a much more realistically sized system.

The first-principles calculations are performed within density functional theory [11, and the local density approximation (LDA) is employed to describe electronelectron interactions. Nonlocal corrections to the exchange-correlation functional have been shown not to have a significant influence on structural and vibrational properties in this system [12]. Optimized pseudopotentials [13] are used to model electron-ion interactions. The one-electron wave functions are expanded in a wellconverged plane-wave basis containing all plane waves up to a 50 Ry cutoff energy. Integrations over the surface Brillouin zone are performed on a discrete mesh of $8 \mathrm{k}$ points with $0.3 \mathrm{eV}$ Gaussian broadening of energy levels.

An analysis of the vibrational states of $\mathrm{CO}$ on copper requires first determining the equilibrium atomic positions. These calculations are summarized in Table 1 . The $\mathrm{O}-\mathrm{C}$ and $\mathrm{C}-\mathrm{Cu}$ bond lengths for the adsorbed molecule are in excellent agreement with experiment [14]. For comparison, the structures of a bare copper (100) surface and a free CO molecule are also presented. At an adsorption site, the copper atom relaxes outward relative to the clean surface by about $1.9 \%$, whereas the empty-site 

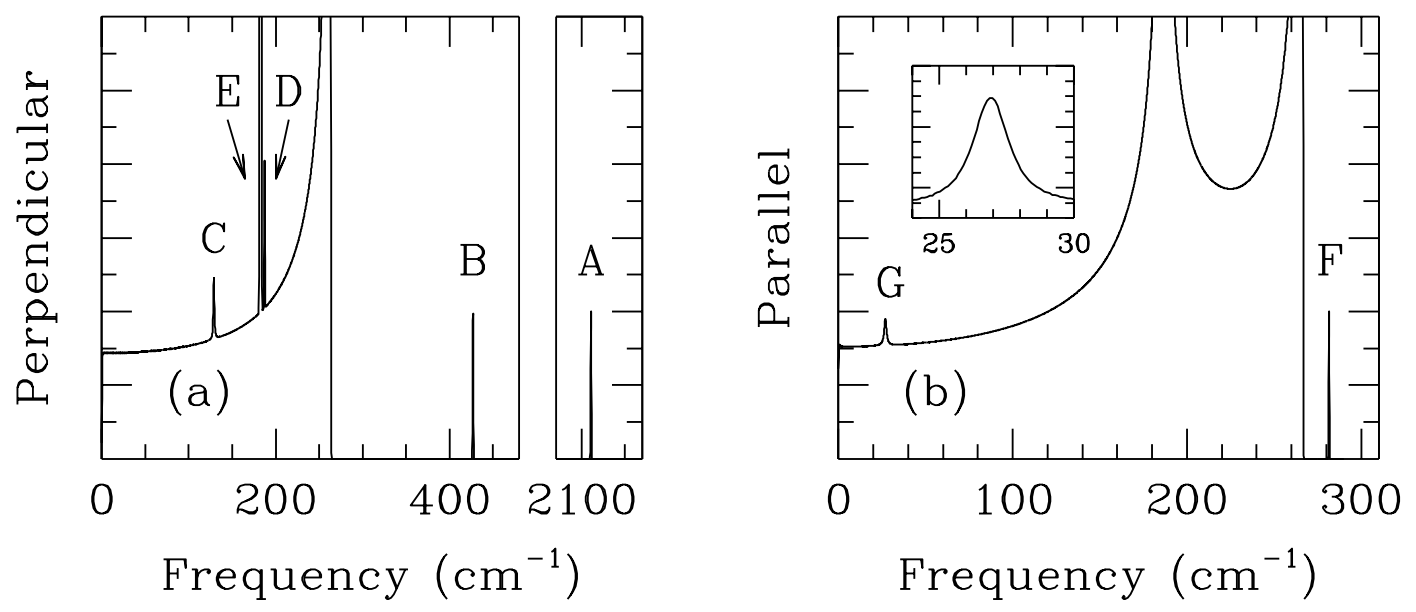

FIG. 1. Density of (a) perpendicular and (b) parallel vibrational modes for a $(\sqrt{2} \times \sqrt{2}) R 45^{\circ}$ overlayer of CO on the copper (100) surface. The inset in (b) highlights the frustrated translational resonance centered at $27 \mathrm{~cm}^{-1}$. The modes correspond to the $\bar{\Gamma}$-point in the surface Brillouin zone. Arbitrary units are used for the density of modes.

coppers remain essentially fixed. Furthermore, the bond length of $\mathrm{CO}$ expands relative to the gas-phase value by about $1.3 \%$.

Vibrational modes at the $\bar{\Gamma}$ point of the $(\sqrt{2} \times \sqrt{2}) R 45^{\circ}$ surface Brillouin zone (SBZ) have been computed and are illustrated in Fig. 1. The spectra are rich in detail, and are in good quantitative agreement with the available experimental data. This serves as strong evidence of the validity of local density functional theory for describing the vibrational properties of this system accurately. Symmetry allows the modes to be classified as having atomic motions either perpendicular or parallel to the surface. The identity of the modes is established by examining the normal-mode polarization vectors. The density of perpendicular modes is shown in Fig. 11(a). It is characterized by a broad band of modes below $263 \mathrm{~cm}^{-1}$ and sharp features labeled $A-E$. The two isolated modes labeled $A$ and $B$ are strongly localized at the adsorbate and constitute the $\mathrm{O}-\mathrm{C}$ and the $\mathrm{C}-\mathrm{Cu}$ bond-stretching modes, respectively. The computed frequencies, $2111 \mathrm{~cm}^{-1}$ and $427 \mathrm{~cm}^{-1}$, respectively, compare favorably with the experimental values of $2085 \mathrm{~cm}^{-1}$ [16] and $345 \mathrm{~cm}^{-1}$ [17.

The features labeled $C$ and $D$ correspond to the surface phonons, $S_{1}$ and $S_{2}$, respectively, at the $\bar{M}$ point of the underlying bare copper SBZ [10]. This point folds back to the zone center in the adsorbed overlayer structure. The $S_{1}$ feature is centered at $129 \mathrm{~cm}^{-1}$, which is in excellent agreement with a helium-atom scattering (HAS) measurement of $123 \mathrm{~cm}^{-1}$ for this system [18]. The $S_{2}$ mode has a frequency of $187 \mathrm{~cm}^{-1}$, which can be compared to the measured bare-surface value of 163 $\mathrm{cm}^{-1} 10$. To our knowledge, no experimental results for this mode have been reported for the adsorbed surface. Finally, the broad band below $263 \mathrm{~cm}^{-1}$ and feature $E$ correspond to bulk copper phonon modes that project onto the $\bar{\Gamma}$ and $\bar{M}$ points, respectively, of the bare SBZ. The latter band is known to be very narrow [19], resulting in a very large peak in the density of modes.

The density of parallel modes is shown in Fig. 1(b). It consists of a broad band below $266 \mathrm{~cm}^{-1}$ and two features labeled $F$ and $G$. The broad band corresponds to bulk copper phonons, with modes below (above) 185 $\mathrm{cm}^{-1}$ coming from the $\bar{\Gamma}(\bar{M})$ point of the bare SBZ. The isolated mode labeled $F$ is highly localized on the adsorbate and corresponds to FR motion of the CO molecule. Its computed frequency is $282 \mathrm{~cm}^{-1}$, in excellent agreement with the $285 \mathrm{~cm}^{-1}$ experimental value [17.

Feature $G$, shown in more detail in the inset of Fig. 1(b), is associated with FT motion of the CO molecule. It contains modes exhibiting the large-amplitude, in-phase wagging motion of carbon and oxygen atoms that is characteristic of frustrated translations. However, an appreciable amplitude is found to persist throughout the substrate, with the copper atoms undergoing bulk phonon motion. The atomic displacements for a typical normal mode in this peak are illustrated in Fig. 2. This behavior demonstrates that FT motion is not a normal mode of CO on copper, but rather it mixes with bulk copper phonons to form a resonance. This resonance has a spectral width of $1.8 \mathrm{~cm}^{-1}$ and is centered at $27 \mathrm{~cm}^{-1}$. HAS measurements find a $\bar{\Gamma}$-point FT frequency of $32 \mathrm{~cm}^{-1}$ for this system [18], indicating excellent agreement between theory and experiment.

The resonant broadening of the FT mode through coupling to bulk phonons provides a purely harmonic description for the observed FT relaxation. In this picture, initial heating of the substrate excites a vibrational wave packet that is localized near the surface. Its normal mode components evolve in time according to different frequencies, causing the wave packet to propagate 


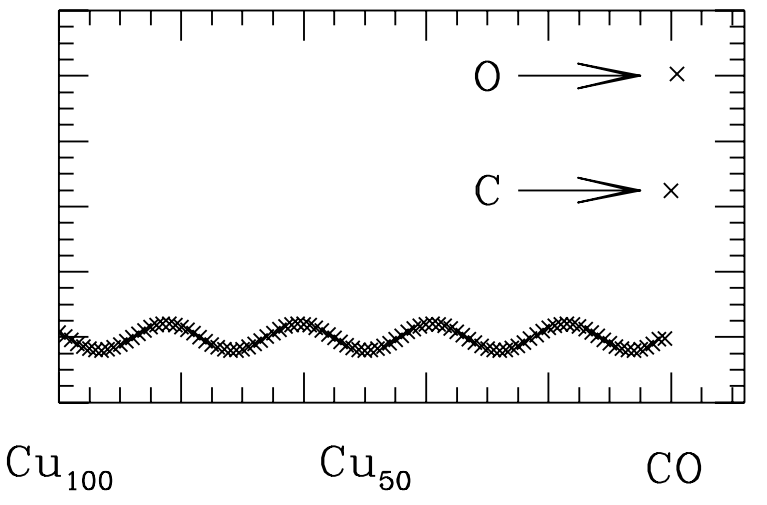

FIG. 2. Illustration of a typical mode in the frustrated translational resonance peak. The vertical axis gives the relative in-plane atomic displacements for the mode, and the horizontal axis labels the atomic layers. Motion of the adsorbate atoms and 100 layers of the copper substrate are shown.

and broaden over time. In this way, vibrational energy initially deposited near the surface spreads throughout the substrate. The spectral width of the FT resonance peak in Fig. 1 (b) gives a measure of the decay rate of the mode and implies a lifetime of $3.0 \mathrm{ps}$. This excellent quantitative agreement with the experimental decay time of $2.3 \pm 0.4 \mathrm{ps}$ at $\mathrm{T} \approx 100 \mathrm{~K}$ is strong evidence that resonant mixing is the dominant mechanism of FT relaxation in this system. This conclusion is consistent with the theoretical work of Ref. [8], which finds that FT decay via nonadiabatic electronic coupling is quite slow for this system $(\tau=108 \mathrm{ps})$.

In this density-functional study, we have explored the vibrational coupling between a $\mathrm{CO}$ adsorbate overlayer and a copper substrate lattice. By treating all atoms of the combined system on an equal footing and including enough bulk copper to describe long-wavelength substrate phonons, we have shown that the vibrational properties of this system are significantly affected by adsorbate-substrate interactions. In particular, we find that the FT mode mixes with long-wavelength, transverse phonons of the substrate to form a resonance. This resonant mixing provides a microscopic description of FT relaxation involving propagation of a wave-packet of harmonic modes. Based on this mechanism, we predict a decay time of $3.0 \mathrm{ps}$, in excellent quantitative agreement with experiment. We thus conclude that harmonic resonant mixing with substrate phonons is the dominant mechanism of FT damping in this system. These calculations highlight the need for a sophisticated treatment of the substrate in achieving a quantitative understanding of the properties of adsorbed metal surfaces.

The authors would like to acknowledge valuable discussions with E. J. Mele, H.-L. Dai, J. P. Culver, and R. M. Hochstrasser. Computational support for this project was provided by the San Diego Supercomputer Center.

[1] G. A. Somorjai, Introduction to Surface Chemistry and Catalysis, (Wiley, New York, 1994).

[2] R. Vanselow, et al., eds. Chemistry and Physics of Solid Surfaces, Vol. I-III, (CRC, Boca Raton, 1977-82); and R. Vanselow and R. Howe, eds. Chemistry and Physics of Solid Surfaces, Vol. IV-VIII, (Springer-Verlag, Berlin, 1982-90).

[3] Various articles in Surf. Sci. 299/300, (1994).

[4] T. A. Germer, J. C. Stephenson, E. J. Heilweil, and R. R. Cavanagh, Phys. Rev. Lett. 71, 3327 (1993); and J. Chem. Phys. 101, 1704 (1994).

[5] J. P. Culver, M. Li, L. G. Jahn, R. M. Hochstrasser, and A. G. Yodh, Chem. Phys. Lett. 214, 431 (1993); and J. P. Culver, M. Li, Z.-J. Sun, R. M. Hochstrasser, and A. G. Yodh, Chem. Phys. 205, 159 (1996).

[6] A. Graham, F. Hofmann, and J. P. Toennies, J. Chem. Phys. 104, 5311 (1996).

[7] B. N. J. Persson and R. Ryberg, Phys. Rev. B 32, 3586 (1985).

[8] M. Head-Gordon and J. C. Tully, J. Chem. Phys. 96, 3939 (1992), and Phys. Rev. B 46, 1853 (1992).

[9] J. C. Tully, M. Gomez, and M. Head-Gordon, J. Vac. Sci. Technol. A 11, 1914 (1993).

[10] Y. Chen, S. Y. Tong, J.-S. Kim, L. L. Kesmodel, T. Rodach, K. P. Bohnen, and K. M. Ho, Phys. Rev. B 44, 11394 (1991).

[11] M. C. Payne, M. P. Teter, D. C. Allan, T. A. Arias, and J. D. Joannopoulos, Rev. Mod. Phys. 64, 1045 (1992), and references therein.

[12] P. H. T. Philipsen, G. te Velde, and E. J. Baerends, Chem. Phys. Lett. 226, 583 (1994); G. te Velde and E. J. Baerends, Chem. Phys. 177, 399 (1993).

[13] A. M. Rappe, K. M. Rabe, E. Kaxiras, and J. D. Joannopoulos, Phys. Rev. B 41, 1227 (1990).

[14] S. Andersson and J. B. Pendry, Phys. Rev. Lett. 43, 363 (1979); and C. F. McConville, D. P. Woodruff, K. C. Prince, G. Paolucci, V. Chab, M. Surman, and A. M. Bradshaw, Surf. Sci. 166, 221 (1986).

[15] Th. Rodach, K.-P. Bohnen, and K. M. Ho, Surf. Sci. 286, 66 (1993).

[16] R. Ryberg, Surf. Sci. 114, 627 (1982).

[17] C. J. Hirschmugl, G. P. Williams, F. M. Hoffmann, and Y. J. Chabal, Phys. Rev. Lett. 65, 480 (1990); J. Electron Spectrosc. 54/55, 109 (1990); and C. J. Hirschmugl, Y. J. Chabal, F. M. Hoffman, and G. P. Williams, J. Vac. Sci. Technol. A 12, 2229 (1994).

[18] J. Ellis, J. P. Toennies, and G. Witte, J. Chem. Phys. 102, 5059 (1995); and M. Bertino, J. Ellis, F. Hofmann, J. P. Toennies, and J. R. Manson, Phys. Rev. Lett. 73, 605 (1994).

[19] R. M. Nicklow, G. Gilat, H. G. Smith, L. J. Raubenheimer, and M. K. Wilkinson, Phys. Rev. 164, 922 (1967). 